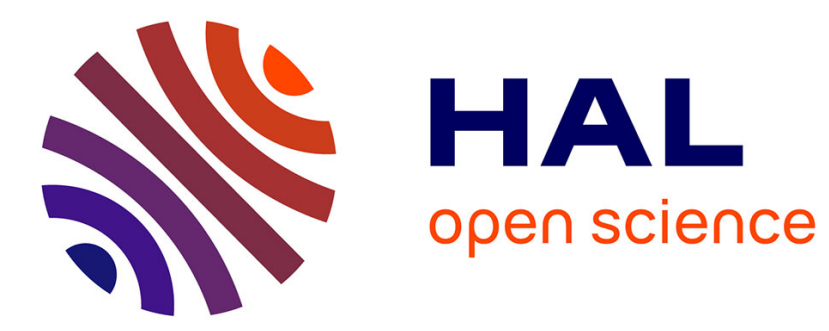

\title{
Optimising TCP over cognitive radio networks for trains
}

Kamal Deep Singh, Jean-Marie Bonnin, David Ros Sanchez

\section{To cite this version:}

Kamal Deep Singh, Jean-Marie Bonnin, David Ros Sanchez. Optimising TCP over cognitive radio networks for trains. ITST 2012: 12th International Conference on ITS Telecommunications, Nov 2012, Taipei, Taiwan. hal-00797550

\section{HAL Id: hal-00797550 https://hal.science/hal-00797550}

Submitted on 6 Mar 2013

HAL is a multi-disciplinary open access archive for the deposit and dissemination of scientific research documents, whether they are published or not. The documents may come from teaching and research institutions in France or abroad, or from public or private research centers.
L'archive ouverte pluridisciplinaire HAL, est destinée au dépôt et à la diffusion de documents scientifiques de niveau recherche, publiés ou non, émanant des établissements d'enseignement et de recherche français ou étrangers, des laboratoires publics ou privés. 


\title{
Optimising TCP over cognitive radio networks for trains
}

\author{
Kamal Deep Singh, Jean-Marie Bonnin and David Ros \\ TELECOM Bretagne, Rue de la Châtaigneraie, 35576 Cesson Sévigné cedex, France \\ Email: \{kamal.singh, jmbonnin, david.ros\}@ telecom-bretagne.eu
}

\begin{abstract}
The Transmission Control Protocol (TCP) is a very popular transport protocol used by the majority of applications. However TCP is inefficient when used over mobile networks. Moreover mobile networks technology is moving towards a new emerging paradigm called Cognitive Radio (CR). Cognitive Radio brings its own share of problems for TCP however more importantly it brings the capability to gather more knowledge about its radio environment. TCP will face same problems, if not more, when used over mobile networks enabled with CR technology. However, these new CR capabilities open the door for better cross layer TCP optimisation techniques. This paper proposes a cross layer distributed mechanism using CR triggers and freeze TCP to optimise TCP over cognitive radio networks for trains. The results show that our mechanism can improve the link utilisation efficiency as compared to standard TCP.

Index Terms-TCP, Congestion control, Cognitive radio
\end{abstract}

\section{INTRODUCTION}

Today, the Transmission Control Protocol (TCP) carries the vast majority of users' traffic, both in wired and wireless networks. TCP transports more than $90 \%$ of the data over the Internet, and it is used by applications as diverse as the web, electronic mail, audio and video streaming (e.g., Spotify, YouTube), instant messaging, peer-to-peer file sharing, and even audio- and video-conferencing (e.g., Skype). Moreover, TCP's share of Internet traffic has been remarkably stable over the last decade, despite changes in applications' popularity. TCP has proved to be very robust in the face of changes in networking technology; indeed, TCP was designed in the 1980's, yet several of its most fundamental features have changed little since. However, it is well known that TCP performance - and, thus, the performance of applications running over TCP - can be very sensitive to the characteristics of the underlying communication links.

Many proposals have been made to improve TCP in general, and to better adapt it to some specific link technologies. In particular, there have been numerous studies of the behavior of TCP over wireless networks. However, cognitive-radio (CR) networks and dynamic spectrum allocation techniques present a new set of challenges to TCP, and in general to layer 4 protocols. An open question is whether existing TCP versions and enhancements may work well in a CR setting.

In this paper, a cross layer distributed mechanism using CR triggers and Freeze TCP [5] to optimise TCP over cognitive

This work was supported by the CORRIDOR project (ANR-11-VPTT-004) of the French national research organisation (ANR). radio networks for trains is proposed. Freeze TCP freezes the TCP sender and its timers by advertising a window size of zero to avoid TCP retransmission timer timeout during temporary disconnections or network problems. This enables Freeze TCP to avoid performance degradation due to problems caused by mobility. This paper is organised as follows. Section II discusses background and related work. Section III proposes the mechanism to optimise TCP over cognitive radio networks for trains. Performance evaluation of our proposal is done in Section IV and Section V concludes this paper.

\section{RELATED WORK AND BACKGROUND}

\section{A. Cognitive radio}

Cognitive radio (CR) [7] is an emerging paradigm for wireless communications and wireless networking, which is being touted as the solution to the above problem of spectrum scarcity. The basic idea of CR-based communications is to use the radio spectrum in an opportunistic manner, while respecting some "etiquette" rules to share the spectrum. CRenabled devices constantly monitor their radio environment in order to find so-called spectrum holes, that is, frequency bands which are unused at a particular time and location. Such bands are then employed for communication between CR devices. Since CR users are likely non-licensed users, they have also to constantly monitor the used band to detect any activity by primary users (i.e., licensed users); as soon as primary users "appear", CR users have to take measures to avoid interfering with them, like e.g. moving to a different, free frequency band or reducing their transmission power.

Cognitive radio is starting to leave the realms of both policy discussions (e.g., the debate on "TV white spaces") and academic research, to go into actual applications. This is attested by related activities in standardization forums like IEEE and ETSI; both recently-completed and draft industry standards, namely: IEEE 802.16h [10] and IEEE 802.11af [9], aim at introducing CR features into wireless regional, metropolitan and local-area networks, respectively. It is therefore of the utmost importance to understand the performance of CR systems, and especially the impact they may have on the performance of users' applications and users' Quality of Experience (QoE). This is especially so since the bulk of the research done in this space has been devoted to the workings of CR systems themselves, with little regard for the users' applications and protocols running on top of them. 
Cognitive radio can be regarded as a natural extension of software-defined radio (SDR), where a so-called cognition cycle allows for wireless terminals to be "aware" of their radio environment and to adapt to it accordingly [11]. Besides some issues shared by all wireless technologies (like e.g. frequent packet losses not due to congestion), new features proper to CR networks like spectrum sensing and spectrum mobility (i.e., switching to a different spectrum band) may have a strong impact on the upper layers of the protocol stack.

\section{B. TCP over Cognitive Radio networks}

TCP is inefficient when used in mobile networks [1] and more so in CR networks for the following reasons [3]:

- Channels with variable characteristics: CR-enabled devices constantly monitor their radio environment to find spectrum holes that can be used. However each channel can have different characteristics in terms of bandwidth, loss and delay. TCP can be slow to adapt to frequent changes in the bandwidth and is sensitive to loss and delay leading to efficiency deterioration.

- Temporary disconnections due to spectrum sensing: Devices with $\mathrm{CR}$ capabilities alternate between sensing mode and transmission mode. During sensing mode, the devices do not transmit the data and this can lead to sudden increase in round trip time $(r t t)$. If TCP's RTO timer $T_{R T O}<r t t+T_{o}$ then timeouts will occur during spectrum sensing period and TCP will set its congestion window (cwnd) to 1 and slow start threshold (ssthresh) is reduced to half of the previous value and TCP enters into the slow-start phase. This leads to the under-utilisation of the available bandwidth as shown in [4].

- Spectrum handover when primary users appear: Whenever a primary user appears in a channel then secondary users have to vacate the channel and have to find another channel to use. In order to transit from one channel to another, the delay and the disconnection period involved can degrade TCP performance and also cause TCP round trip timer timer to expire reducing its efficiency.

\section{Related Work}

Since its introduction by Mitola [11], the concept of cognitive radio has been the subject of much research effort. The vast majority of the published studies and proposals are related to the physical layer (with topics like primary-user detection being the focus of a good deal of papers), as well as to the link layer. Moreover, some work has also been devoted to network-layer. However, to the best of our knowledge, the relation and interactions between cognitive radio and the upper protocol layers have not received much attention by the research community. In particular, there is little published work on transport-layer issues in a cognitive radio setting, with only a couple of papers [12], [13] considering the multihop case at all, and the other studies focusing on simplistic singlehop scenarios [3], [14]. Besides, there exist just a handful of proposals dealing with improving transport protocol performance, either by means of cross-layer optimization [15], by optimizing link layer parameters such as frame size for cognitive radio networks [16], through new protocol proposals [2] or by integrating an existing protocol into a cognitive framework [17]. It is however crucial to understand how such end-to-end protocols and mechanisms would work over CR systems.

TCP problems in CR networks are also discussed in [6]. Some receiver based TCP improvement solutions to tackle problems due to wireless conditions and mobility are discussed in [8] such as:

- DelAck: An ACK for every d packet or send an ACK if a segment has been unacknowledged for more than $\mathrm{T}$ seconds (eg. 0.1s)

- TCP-ADA (TCP adaptive delay acknowledgment) postpones ACKs for a time period in proportion to exponential moving average of inter arrival time between successive segments. Deferment timer is restarted every time a new segment arrives before timeout. But can lead to loss of ACK clocking.

Besides, some proposals for coping with transport-layer issues in wireless networks could perhaps be useful in CR networks - such as the work in [5] that proposes an end-toend way to freeze TCP source to tackle disconnections due to mobility and handover. It is however crucial to understand how such end-to-end protocols and mechanisms would work over CR systems.

\section{OPtimising TCP OVER COGNITIVE RADiO NETWORKS FOR TRAINS}

In order to improve TCP over Cognitive radio network for trains, this paper proposes a generic and practical solution using cross layer techniques. The goal is to improve TCP's performance when faced with mobility and wireless conditions. Moreover this optimisation should ensure that there is no need to modify all the routers and hosts in the Internet. For this the new solution should be perfectly compatible with standard TCP.

This paper proposes to implement CR capabilities in the mobile router that will provide connectivity to the clients present in the train. These CR capabilities will allow the Mobile router to predict a disconnection and the link layer will send triggers warning the clients about a pending disconnection as shown in Figure 1.

A small connection manager application will be installed in the clients that will reside in the application layer. This application will also manage TCP connections and our solution will not modify anything in the TCP stack. It is already a common practice to install a small software in the clients before Internet connectivity is provided to them, for example, when using 3G USB keys. Thus, installing this application will not degrade the user experience. 


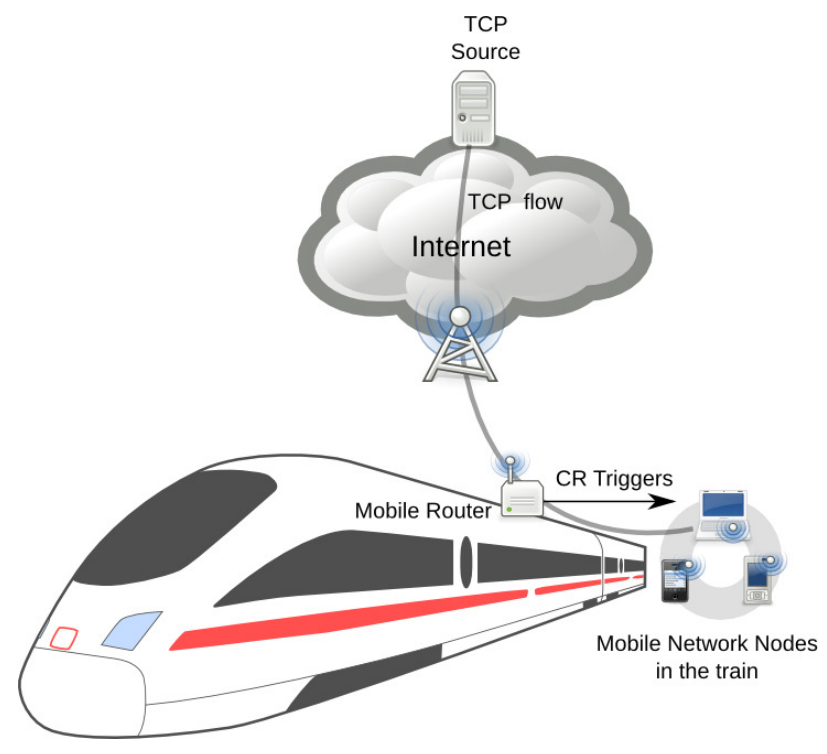

Fig. 1. CR triggers sent by Mobile router.

\section{A. CR triggers}

There are two types of CR triggers that are needed from the mobile router. The first one warns the clients connected to the mobile router about a pending disconnection and the second one informs the clients that the link will start working.

- Link going down

- Link going up

For the downlink traffic, on the reception of "Link going down" trigger, the connection manager sends the freeze signal that sets the receiver window to be zero and thus freezes the TCP source. On receiving "Link going up" it sends triplicate ACKs that unfreeze the TCP source. Freeze TCP uses triplicate ACKs to avoid sender being idle even after the link is up. For more details please see [5]. For the uplink traffic, the connection manager directly freezes or unfreezes the TCP source on the reception of the above respective triggers.

Detection of disconnection start and duration is needed in advance such that CR triggers can arrive in advance. If the first trigger is late then the link will go down before the connection manager can react and if the trigger is early then the TCP source will freeze and stop sending data even when the link is available. Both the cases will not be optimal for TCP performance. As shown in Figure 2 the triggers have to arrive at least $T U_{\text {advance }}$ time before a link goes down. From Figure 2, and as shown later in section IV, it can be seen that optimal value of $T U_{\text {advance }}$ is given as follows:

$$
\begin{gathered}
T U_{\text {advance }}=R T T_{\text {local }}+R T T_{\text {sender }} \\
=R T T_{T C P} .
\end{gathered}
$$

The trigger "Link going up" has to be sent in advance too such that when the link is up the triplicate ACKs should arrive to the TCP source as soon as possible. From Figure 2

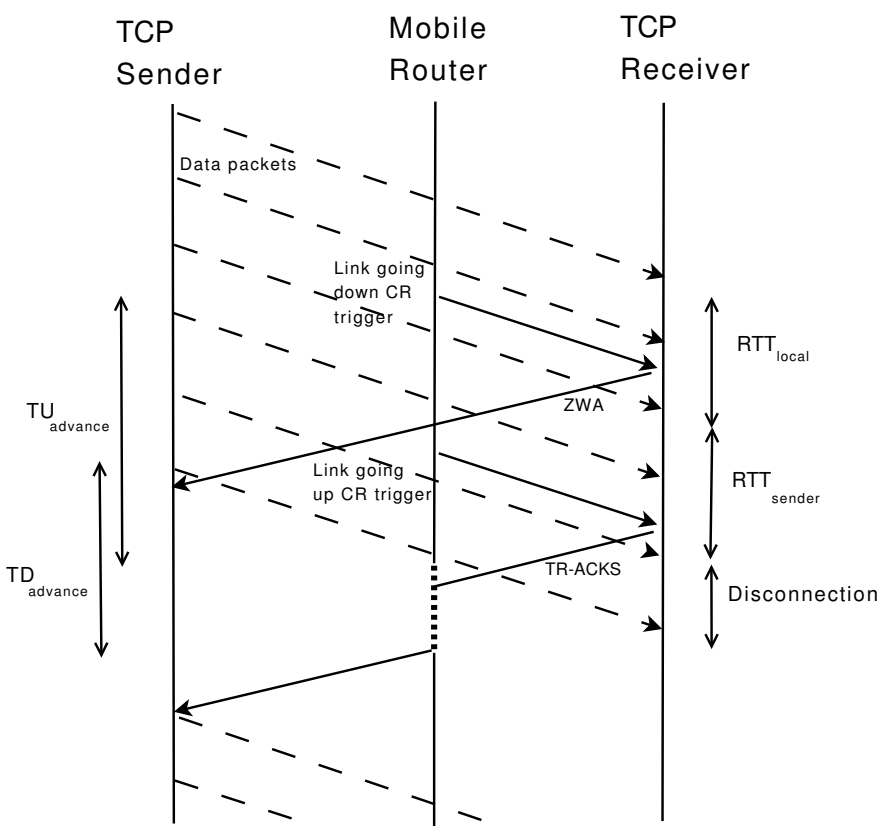

Fig. 2. Delay diagram for CR triggers.

it can be seen any value of $T D_{\text {advance }}$ such that:

$$
R T T_{\text {local }}<T D_{\text {advance }}<R T T_{\text {local }}+D
$$

with $D$ as the disconnection duration, will be optimal if the mobile router can store the arriving triplicate ACKs during disconnection and later send them as soon as the link is up.

Thus, Mobile router needs to know the values of $R T T_{T C P}$ and $R T T_{\text {local }}$. Moreover, these values will be different for different clients connected to it. It will obtain the values of $R T T_{T C P}$ values for all the clients from the connection manager installed in those clients as each connection manager installed on the clients monitors the TCP connections of that client. The connection manager will monitor the value of $R T T_{T C P}$ and will send it in response as Mobile router will periodically request for this value. The $R T T_{\text {local }}$ values are estimated by Mobile router by noting the time elapsed between sending a request to the connection manager and the arrival of its response. It should be noted that frequency of such requests should be low to avoid significant overhead. After that Mobile router will use the maximum values of $R T T_{T C P}$ and $R T T_{\text {local }}$ among different clients to calculate the values of $T U_{\text {advance }}$ and $T D_{\text {advance }}$ respectively.

Detection of disconnections and re-connections is done in two ways. Train itineraries are fixed and disconnections are predicted based on geolocalisation and recorded statistics during the previous journeys. In addition CR capabilities based on tracking the signal strength, such as in [19], are used to predict the pending disconnection. For that this paper proposes to implement these above two functions in the mobile router. 


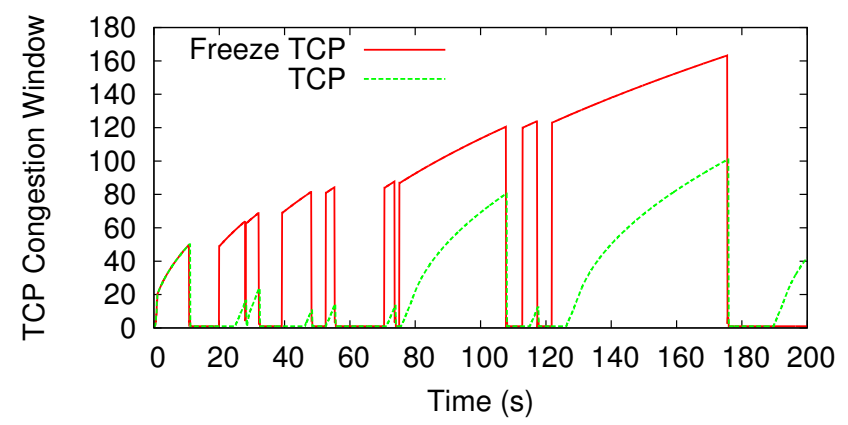

Fig. 3. Congestion window evolution for Freeze TCP and TCP New Reno.

\section{B. Connection manager}

Connection manager software, similar to [18], is installed on a client when it connects to Mobile router for the first time. It does not modify the TCP stack, but it keeps a track of outgoing and incoming TCP packets. When it receives the "Link going down" trigger from Mobile router it sets the receiver window in outgoing ACKs as zero, or zero window advertisement (ZWA), to send the freeze signal to the TCP source. Similarly when it receives the "Link going up" trigger it generates triplicate ACKs (TR-ACKs) to unfreeze the TCP source. The idea is to use advance and precise information on link going up and down using cognitive radio capabilities.

This approach of tracking TCP flows at the client instead of tracking them at Mobile router has the advantage that the approach becomes distributed. Thus, Mobile router doesn't have to track many simultaneous connections passing through it which would have increased the computational load on it. Rather it just keeps the maximum values of $R T T_{T C P}$ and $R T T_{\text {local }}$. As discussed in section III-A, connection manager also monitors the value of $R T T_{T C P}$ for each connection and sends their values when mobile router requests for them.

\section{REsults}

In this section, we study the performance of our proposed scheme using CR triggers and Freeze TCP over cognitive radio networks for trains. The study is done using NS-2. The topology is as shown in Figure 1, the delay between TCP sources and Node B is $50 \mathrm{~ms}$, the delay between NodeB and Mobile router is $30 \mathrm{~ms}$ and the delay between Mobile router and mobile clients present in the train is $10 \mathrm{~ms}$. The link between NodeB and Mobile router has 2Mbps of bandwidth. The default number of mobile clients is 10 unless specified.

Figure 5 shows that the efficiency of link utilisation is best when $T U_{\text {advance }}=R T T_{T C P}$. This was also discussed before in section III. Hence from now onwards let us fix the value of $T U_{\text {advance }}$ to $R T T_{T C P}$ for next simulations.

Disconnections due to handovers and due to train passing through tunnels are simulated using an exponential distribution with average disconnection time of 5 seconds and average up time of 20 seconds. Figure 3 shows the evolution of congestion window of Freeze TCP as compared to normal TCP (New Reno) and for this simulation only 1 mobile

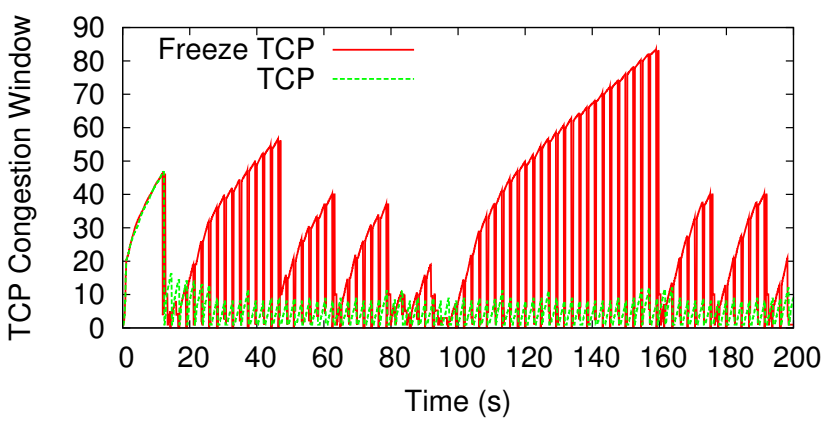

Fig. 4. TCP congestion window evolution when sensing duration $T_{o}$ is 0.3 seconds.

client is connected to the mobile router. It can be seen that congestion window of normal TCP falls every time there is a disconnection and TCP enters into slow start that in turn reduces the link utilisation efficiency that is defined as:

$$
\text { efficiency }=\frac{\text { TotalBitsTransmitted }}{\text { Linkuptime } * \text { LinkCapacity }}
$$

However when Mobile router with CR trigger mechanism is used then the TCP source freezes the congestion window as soon as it receives ZWA and later resumes using the same frozen value of congestion window when disconnection gets over and the efficiency is improved as compared to normal TCP as shown in Figure 6.

Moreover, as discussed before, devices with CR capabilities alternate between sensing mode and transmission mode. During sensing mode, the devices do not transmit the data and this can introduce spikes in round trip times of TCP packets leading to TCP's RTO timer timeouts when sensing duration $T_{o}$ is long for example 0.3 seconds. Figure 7 shows the effect of increasing values of sensing durations. The value of the duration of transmission mode is 2 seconds. The results for $T_{o}$ of up to 0.5 seconds are shown as $T_{o}$ of the order of 0.3 seconds is considered in [2]. It can be seen that total throughput over a link having the capacity of $2 \mathrm{Mbps}$ decreases with increasing $T_{o}$. Our scheme that uses CR triggers and freeze TCP mechanism again shows significant improvement as compared to standard TCP as it freezes TCP source before sensing starts and avoids RTO timeouts. Figure 4 shows the evolution of congestion window for $T_{o}=0.3 \mathrm{~s}$ and it should be noted that for the first 10 seconds the sensing simulation is not started in order to allow TCP to finish the initial slow start phase. As discussed before, with normal TCP, the RTO timer expires when $T_{R T O}<r t t+T_{o}$ and this happens more frequently for higher values of $t_{o}$ thus explaining the degradation of link utilisation efficiency with normal TCP. Whereas relatively better performance can be observed with our scheme that uses CR-triggers and freeze TCP.

\section{CONCLUSions}

In this paper, a mechanism is proposed that uses link layer triggers from cognitive radio modules and freeze TCP to optimise the performance of TCP over cognitive radio 


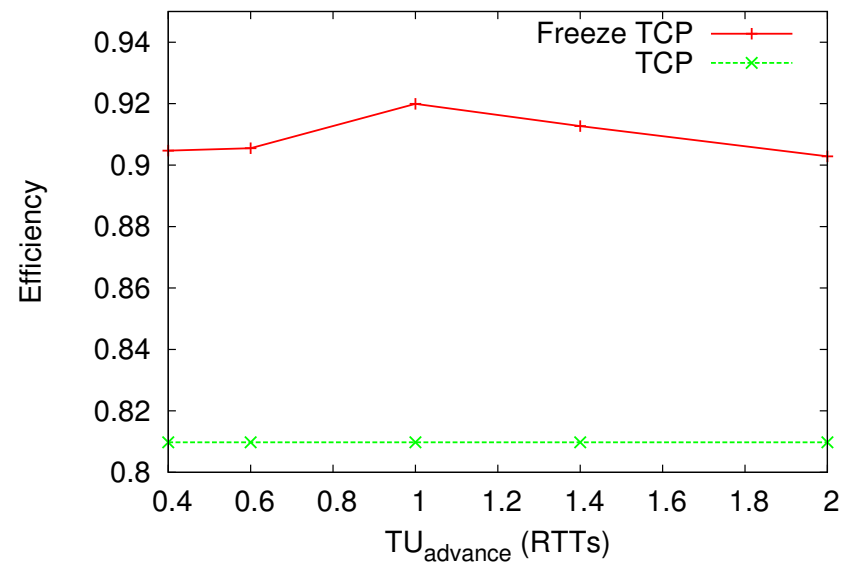

Fig. 5. Efficiency of Link utilisation with respect to different values of $T U_{\text {advance }}$ as a multiple of RTTs.

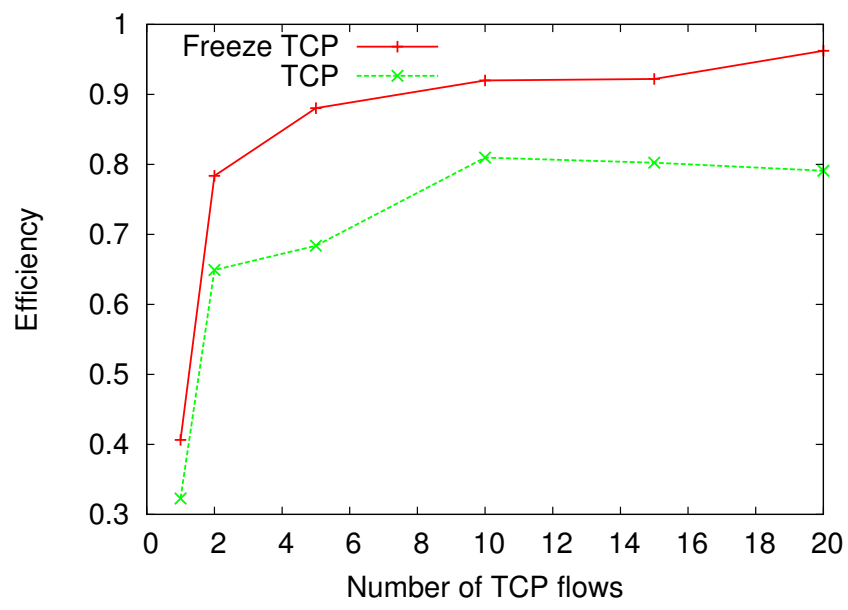

Fig. 6. Efficiency with respect to increasing number of TCP flows.

networks for train. The proposed mechanism uses a cross layer distributed approach in which a connection manager is installed on clients wanting to use Internet connectivity on trains. The connection manager manages and optimises the TCP connection without changing TCP stack and without breaking compatibility with standard TCP implementations. The performance of the proposed mechanism was evaluated and it showed significant improvement in terms of link utilisation efficiency as compared to standard TCP. In future we would like to do develop our simulator with more details related to high speed mobility and additional cognitive radio parameters.

\section{REFERENCES}

[1] Hari Balakrishnan, Venkata N. Padmanabhan, Srinivasan Seshan, Randy H. Katz: "A comparison of mechanisms for improving TCP performance over wireless links.” IEEE/ACM Trans. Netw. 5(6): 756-769 (1997).

[2] K. R. Chowdhury, M. D. Felice and I. F. Akyildiz. "TP-CRAHN: A Transport Protocol for Cognitive Radio Ad-hoc Networks.” Proc. IEEE, INFOCOM 2009, Rio de Janeiro, Brazil, Apr. 2009.

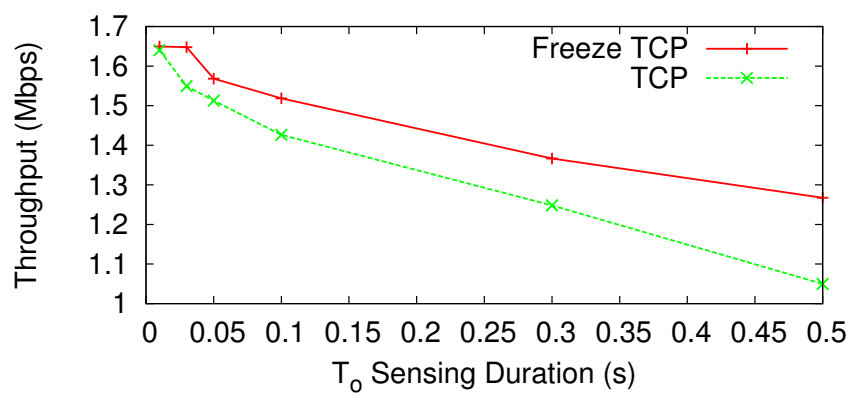

Fig. 7. Throughput with respect to different values of sensing duration $T_{o}$.

[3] Y. R. KondaReddy and P. Agrawal. "Effect of Dynamic Spectrum Access on Transport Control Protocol Performance." IEEE GLOBECOM 2009, Nov. 30 - Dec. 42009.

[4] Dilip Sarkar and Harendra Narayan. "Transport Layer Protocols for Cognitive Networks.", IEEE Conference on Computer Communications Workshops, INFOCOM Workshops, 2010.

[5] T. Goff, J. Moronski, D. S. Phatak and V. Gupta. "Freeze-TCP: A true end-to-end TCP enhancement mechanism for mobile environments" Proc. IEEE, INFOCOM 2000, Tel-Aviv, Israel, Mar. 2000.

[6] Ian F. Akyildiz, Won-Yeol Lee, Mehmet C. Vuran and Shantidev Mohanty, "NeXt generation/dynamic spectrum access/cognitive Radio Wireless Networks: A Survey". COMPUTER NETWORKS JOURNAL (ELSEVIER), vol. 50, pp. 2127-2159, 2006.

[7] S. Haykin, "Cognitive radio: brain-empowered wireless communications", Selected Areas in Communications, IEEE Journal on, Vol. 23 , No. 2. (07 February 2005), pp. 201-220.

[8] Ka-Cheong Leung, Victor O. K. Li, "Transmission control protocol (TCP) in wireless networks: issues, approaches, and challenges", IEEE Communications Surveys \& Tutorials, Vol. 8, No. 4. (4th Quarter 2006), pp. 64-79.

[9] "IEEE P802.11 Task Group af - Wireless LAN in the TV White Space."

[10] "IEEE Std 802.16h-Part 16: Air Interface for BroadbandWireless Access Systems; Amendment 2: Improved Coexistence Mechanisms for License-Exempt Operation", July 2010.

[11] J. Mitola III and G.Q. Maguire Jr. "Cognitive radio: making software radios more personal." IEEE Personal Communications, 6(4):13-18, August 1999.

[12] M. Di Felice, K.R. Chowdhury, and L. Bononi. "Modeling and performance evaluation of Transmission Control Protocol over cognitive radio ad hoc networks." In Proceedings of ACM MSWiM, pages 4-12, Tenerife, October 2009

[13] M. Di Felice, K.R. Chowdhury, W. Kim, and A. Kassler. "End-to-end protocols for cognitive radio ad hoc networks: An evaluation study." Performance Evaluation, in print, 2011.

[14] T. Issariyakul, L.S. Pillutla, and V. Krishnamurthy. "Tuning radio resource in an overlay cognitive radio network for TCP: Greed isn't good. IEEE Communications Magazine," 47(7):57-63, July 2009.

[15] C. Luo, F.R. Yu, H. Ji, and V.C.M. Leung. "Optimal channel access for TCP performance improvement in cognitive radio networks: A cross-layer design approach.” In Proceedings of IEEE GLOBECOM, Honolulu, December 2009.

[16] C. Luo, F.R. Yu, H. Ji, and V.C.M. Leung. "Cross-layer design for TCP performance improvement in cognitive radio networks." IEEE Transactions on Vehicular Technology, 59(5):2485-2495, June 2010.

[17] B.S. Manoj, R.R. Rao, and M. Zorzi. "CogNet: a cognitive complete knowledge network system." IEEE Wireless Communications, 15(6):8188, December 2008.

[18] Rayene Ben Rayana, J-M Bonnin, "Mobility aware application manager for mobile networks." ITST 2008. 8th International Conference on ITS Telecommunications, Pages 337-342.

[19] Yoo, Sang-Jo, David Cypher and Nada Golmie, "LMS Predictive Link Triggering for Seamless Handovers in Heterogeneous Wireless Networks." Military Communications Conference, 2007, IEEE MILCOM 2007, 29-31 Oct. 